\title{
Time-Resolved Fluorescence Anisotropy Study on Intramolecular Interactions of Branched Styryl Derivatives Based On 1, 3, 5-Triazine
}

\author{
Y.C. Wang \\ Department of Physics \\ Dalian Maritime University \\ Dalian, China \\ *Corresponding author \\ G.Q. Wang \\ Department of Physics \\ Dalian Maritime University \\ Dalian, China
}

\author{
D.J. Liu \\ Department of Physics \\ Dalian Maritime University \\ Dalian, China \\ *Corresponding author
}

B. $\mathrm{Li}$

Department of Electronic Engineering

East China Normal University

Shanghai, China

*Corresponding author

\begin{abstract}
A series of branched styryl derivatives based on 1, 3, 5-triazine have been studied by time-resolved fluorescence anisotropy method to study the intramolecules interferaction between branches. The obtained results further confirmed the TPA enhancement mechanism, the anisotropy of trimer shows faster decay and small residual value indicates there are strong intramolecules interactions among branches, this maybe the enhancement mechanism of TPA properties for the trimer.
\end{abstract}

Keywords-two photon absorption; fluorescence; time-resolved fluorescence anisotropy

\section{INTRODUCTION}

Materials with large two-photon absorption (TPA) properties are of great interest in many fields [1-3], such as optical limiting, three-dimensional microfabrication, optical storage, and so on. Design and synthesis of the organic and/or polymeric functional materials with excellent TPA performance have thus stimulated extensive research activities across the world in recent years [4].

Although there already are a large number of papers and patents concerning preparation of TPA materials, including one-dimensional dipolar [5], quadrupolar [6], and multibranched chromophores [7], development of the molecular materials with large TPA cross section still draws much attention and presents an ongoing challenge.

To design and synthesize more excellent molecules with outstanding TPA properties, a proper understanding of the dynamics of two-photon excitation process in the materials is of great importance. Femtosecond (fs) pump-probe experiment and time-resolved fluorescence and time-resolved fluorescence anisotropy are very useful technique to obtain the information of the relaxation processes of the excited states. Theodore Goodson,
III, Varnavski and co-workers et al. employed pump-probe, time-resolved photoluminescence, and three-pulse photon echo measurements to measure dynamics of some molecules including multibranched chromophores and organic conjugated dendrimers [8-9]. Anisotropy study is a powerful tool to give additional information about the energy redistribution and the dynamics of electronic coupling in multi-branched molecules. It has been widely used in experiments on photosynthetic reaction center. Goodson et al. reported plenty of significant results based on time-resolved fluorescence up-conversion measurement on optical dendrimers[10-11]. Dynamics of molecules with two-photon absorption properties are, however, still inadequate especially for the conjugated molecules. In our previous work, we reported the TPA character and excited state dynamics of several molecules and polymers with both linear and tri-branched structure [12-14]. Recently, we investigated a tri-branched materials, by using time-resolved fluorescence anisotropy methods, The tri-branched materials displays very good two photon absorption ability and nonlinear enhancement according to monomer case. The time-resolved photoluminescence anisotropy results indicate the enhancement mechanism of TPA properties.

\section{MATERIALS AND EXPERIMENTAL METHODS}

The structures of T01, T02 and T03 are shown in Figure 1. The synthesis method, UV-visible absorption spectra, fluorescence as well as TPF/TPA properties have been reported elsewhere in detail [15]. Two-photon absorption (2PA) cross-sections measured by the open aperture Z-scan technique were determined to be 77, 90 and $410 \mathrm{GM}$ for T01, T02 and T03, respectively. 


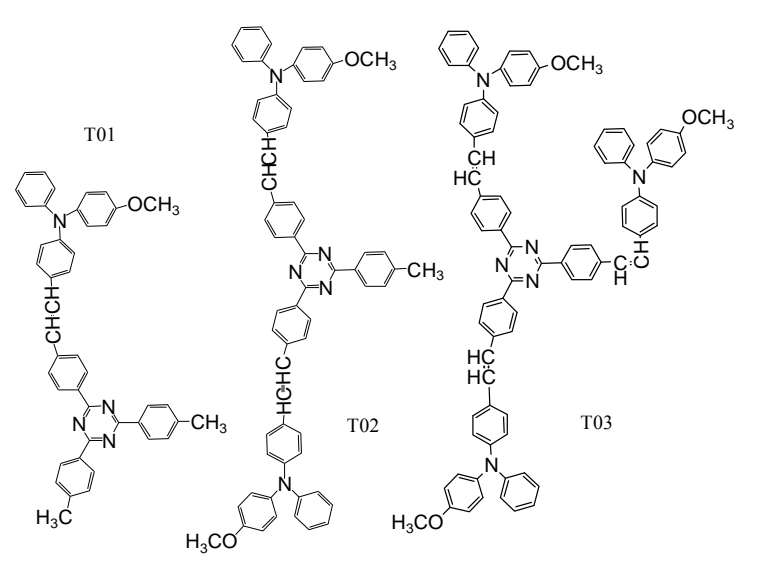

FIGURE I. THE STRUCTURE OF T01, T02 AND T03.

The ultrafast responses of the polymer were investigated by fs time-resolved photoluminescence (TRPL) experiments. The setup is shown in Figure 2. Briefly, the pump beam at $800 \mathrm{~nm}$ after passing an optical delay line was used as a gate beam to open "Kerr gate" through photo-induced birefringence of Kerr Material (CS2), while the second part of $800 \mathrm{~nm}$ beam was frequency doubled by using a BBO crystal to act as the probe beam. The second harmonic pulses with vertical polarization were used to pump samples efficiently and the collected fluorescence was set either parallel or perpendicular to that of incident beam by a polarizer (P1). Another polarizer with orthogonal polarization to P1 was placed after sample to study the polarization effect. Dispersed by a monochromator, the signal was detected by a photomultiplier (Hamamatsu R1104) connected to a lock-in amplifier (SR830, Stanford Research Systems). The polarization of the gate beam was set at $45^{\circ}$ with respect to that of SHG. In doing so, we can get TRFL signals under different configuration for anisotropy study.

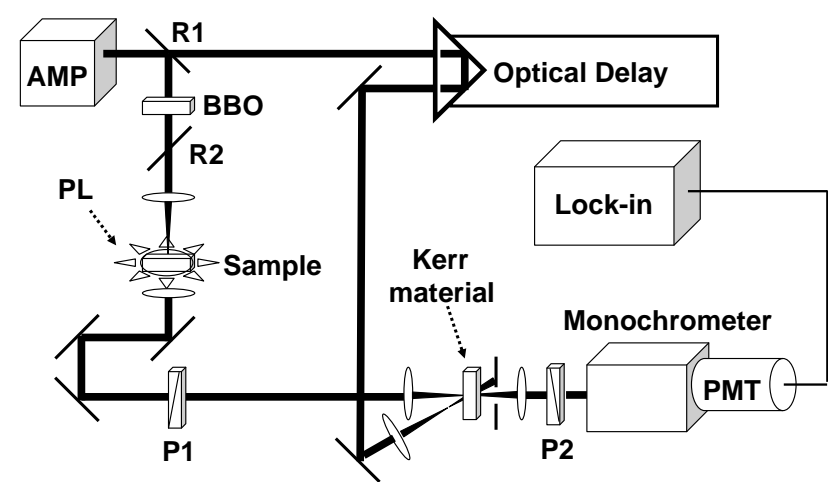

FIGURE II. EXPERIMENTAL SETUP FOR THE OKG METHOD. R1: BEAM SPLITTER; R2: HIGH REFLECTIVE MIRROR AT 800 NM; P1 AND P2: POLARIZERS (CROSS-POLARIZATION); KERR MATERIAL: CS2 IN 5 MM CELL; PMT: PHOTOMULTIPLIER TUBE.

The fs pulses employed in ultrafast dynamics measurements were generated by amplification stage of the used fs laser system (Spitfire, Spectra-Physics). The average output power from the Spitfire was about $300 \mathrm{~mW}$. The pulse duration was $140 \mathrm{fs}$, the wavelength was $800 \mathrm{~nm}$ and the repetition rate was $1 \mathrm{kHz}$. In the experimental investigation, the solvent $\mathrm{CHCl} 3$ has been used without further distillation. All the experiments were carried out at room temperature.

\section{RESULTS AND DisCUSSIONS}

As we all know, anisotropy can be decided by

$$
r(\tau)=\frac{I_{/ /}-I_{\perp}}{I_{/ /}+2 I_{\perp}}
$$

Where $I_{/ /}\left(I_{\perp}\right)$ denote the fluorescence intensity whose polarization is parallel (perpendicular) to that of excited beam. However, analyzing TRPL experimental data based on OKG technique is a great challenge because it is more complicated than the analysis of transient absorption and fluorescence up-conversion experimental data. In the latter, the system response can be simply considered as Gaussian which is convenient for deconvolution treatment. In our experiment, the system response should be the response profile of CS2, which is nearly exponential type. Thus we used the following two formulas to calculate:

$$
I_{/ /}=\int \sigma(t-\tau)\left[A_{1} e^{-t / \tau_{1}}+A_{2} e^{-t / \tau_{2}}+A_{3} e^{-t / \tau_{3}}\right]\left[1+2\left\{\left(r_{0}-r_{1}\right) e^{-t / \tau_{r}}+r_{1}\right\}\right] d t
$$

$$
I_{\perp}=\int \sigma(t-\tau)\left[A_{1} e^{-t / \tau_{1}}+A_{2} e^{-t / \tau_{2}}+A_{3} e^{-t / \tau_{3}}\right]\left[1-\left\{\left(r_{0}-r_{1}\right) e^{-t / \tau_{r}}+r_{1}\right\}\right] d t
$$

Where $\tau_{r}$ is the time constant of anisotropy decay, ${ }^{r}$ and $r_{1}$ represent initial value and residual value, respectively.

Figure 3 shows the TRFL anisotropy experimental results of T01, T02, and T03 in THF solution at $510 \mathrm{~nm}$ and $550 \mathrm{~nm}$, respectively. The fitting results are summarized in Table I. It can be seen from the results that, there is a fast anisotropy decay process at wavelength of $510 \mathrm{~nm}$ for all the three compounds. The lifetimes of the process are $\sim 500 \mathrm{fs}, 415 \mathrm{fs}, 350 \mathrm{fs}$ for monomer, dimmer and trimer, respectively. The initial anisotropy value and resistant value is 0.65 and 0.46 for monomer T01. For dimmer T02, the initial anisotropy value and residual value is 0.57 and 0.43 . Trimer T03 hold the smallest initial anisotropy value and residual value $(0.52 / 0.36)$, the more branches, the smaller two values. When the probe wavelength tuned to $550 \mathrm{~nm}$, no obvious anisotropy decay process was observed for all three compounds. The two values remain at some value. The value is also decrease with increase of number of branches. Usually, large initial anisotropy value as well as fast decay indicates strong intramolecular interactions which directly affect energy redistributions and bring large nonlinear optical effect [10]. This may be a reason why TPA cross section of trimer T03 is is about 5.32-fold larger than that of monomer T01, the anisotropy decay time is shorter than that of monomer T01. 
TABLE I. THE TIME-RESOLVED FLUORESCENCE ANISOTROPY RESULTS OF MONOMER, DIMER AND TRIMER T01, T02 AND T03.

\begin{tabular}{|c|c|c|c|c|}
\hline & $\mathrm{T}(\mathrm{fs})$ & $\mathrm{R} 1$ & $\mathrm{R} 0$ & $\mathrm{R} 550 \mathrm{~nm}$ \\
\hline $\mathrm{T} 01$ & $\approx 500$ & 0.65 & 0.46 & 0.4 \\
\hline $\mathrm{T} 02$ & $\approx 415$ & 0.57 & 0.43 & 0.38 \\
\hline $\mathrm{T} 03$ & $\approx 350$ & 0.52 & 0.36 & 0.2 \\
\hline
\end{tabular}

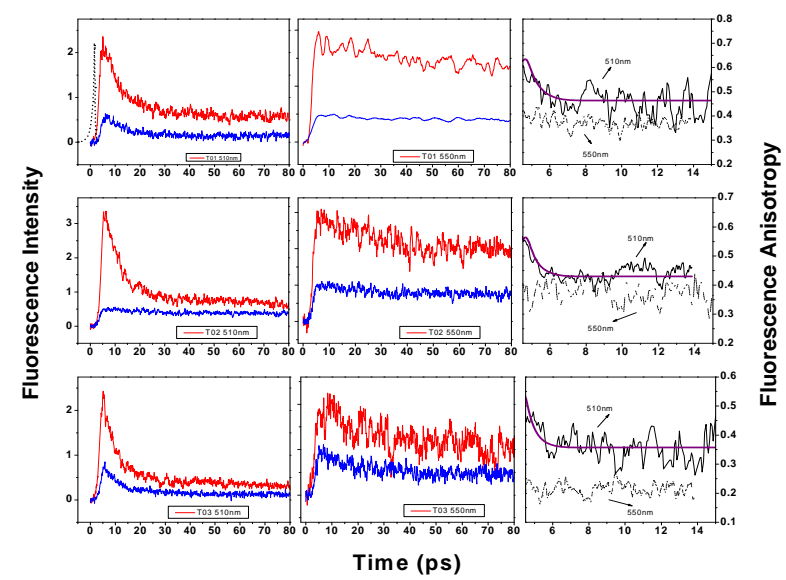

FIGURE III. EXPERIMENTAL SETUP FOR THE OKG METHOD. R1: BEAM SPLITTER; R2: HIGH REFLECTIVE MIRROR AT 800 NM; P1 AND P2: POLARIZERS (CROSS-POLARIZATION); KERR MATERIAL: CS2 IN 5 MM CELL; PMT: PHOTOMULTIPLIER TUBE.

\section{SUMMARY}

In this study, the anisotropy of monomer T01, dimer T02 and trimer T03, are investigated by time-resolved fluorescence anisotropy technique. Obvious different between monomer, dimer and trimer are observed. It was found that, dimer and trimer show shorter depolarization time and less residual values in comparison with monomer. The anisotropy of trimer shows faster decay and small residual value indicates there are strong intramolecules interactions among branches, this maybe the enhancement mechanism of TPA properties for the trimer.

\section{ACKNOWLEDGEMENT}

We sincerely thank the financial support from National Natural Science Foundation of China (11404048, 61205154, 11375034) and the Fundamental Research Funds for the Central Universities (3132013104, 3132015233, 3132015152, $3132013106,3132014231,3132014337)$ and the program for Liaoning Excellent Talents in University (Grant No. LJQ2014051)

\section{REFERENCES}

[1] J. D. Bhawaolar, G. S. He and P. N. Prasad, Nonlinear multiphoton process in organic polymeric materials, Rep. Prog. Phys. 59(1996) 1041-1070.

[2] Q. D. Zheng, S. K. Gupta, G. S. He, L-S. Tan, P. N. Prasad, Synthesis, Characterization, Two-Photon Absorption, and Optical Limiting Properties of Ladder-Type Oligo-p-phenylene-Cored Chromophores, Adv. Funct. Mater. 18(2008)2770-2779.

[3] W. Denk, J. H. Strickler, and W. W. Webb, Two-photon Laser Scanning Fluorescence Microscopy, Science. 248(1990) 73-76.
[4] M. Albota, D. Beljonne, J. L. Bredas, J. E. Ehrlich, J. Y. Fu, A. A. Heikal, S. E. Hess, T. Kogej, M. D. Levin, S. R. Marder, D. McCord-Maughon, J. W. Peny, H. R6ckel, M. Rumi, G. Subramaniam, W. W. Webb, X. L. Wu and C. Xu, Design of Organic Molecules with Large Two-Photon Absorption Cross Section, Science 281(1998) 1653-1656.

[5] B. A. Reinhardt, L. L. Brott, J. J. Clarson, Highly active two-photon dyes: design, synthesis and characterization toward applicatioin, Chem. Mater. 10(1998)1863-1874.

[6] M. Albota, D. Beljonne, J. L. Bredas, J. E. Ehrlich, J. Y. Fu, A. A. Heikal, S. E. Hess, T. Kogej, M. D. Levin, S. R. Marder, D. McCord-Maughon, J. W. Peny, H. R6ckel, M. Rumi, G. Subramaniam, W. W. Webb, X. L. Wu and C. Xu, Design of Organic Molecules with Large Two-Photon Absorption Cross Section, Science 281(1998) 1653-1656.

[7] T.-C. Lin, Y.-F. Chen, C.-L. Hu, C.-S. Hsu, Two-photon absorption and optical power limiting properties in femtosecond regime of novel multi-branched chromophores based on tri-substituted olefinic scaffolds, J. Mater. Chem. 19(2009)7075-7080.

[8] M. I. Ranasinghe, Y. Wang, T. III. Goodson, Excitation energy transfer in branched dendritic macromolecules at low (4K) temperatures, J. Am. Chem. Soc. 125(2003) 5258.

[9] Y. Wang, G. S. He, P. N. Prasad, T. Goodson III, Ultrafast Dynamics in Multibranched Structures with Enhanced Two-Photon Absorption, J. Am. Chem. Soc. 127(2005) 10128-10129.

[10] O. P. Varnavski, J. C. Ostrowski, L. Sukhomlinova, R. J. Twieg, G. C. Bazan, T. Goodson III, Coherent Effects in Energy Transport in Model Dendritic Structures Investigated by Ultrafast Fluorescence Anisotropy Spectroscopy, J. Am. Chem. Soc. 124(2002) 1736-1743.

[11] T. III. Goodson, Optical excitations in organic dendrimers investigated by time-resolved and nonlinear optical spectroscopy, Acc. Chem. Res. 38(2005) 99.

[12] Y. C. Wang, Y. L. Yan, B. Li and S. X. Qian, The study on the two-photon absorption/fluorescence properties and the ultrafast dynamics of the organic materials, Progress In Physics. 32(2012) 1-30.

[13] Y. C. Wang, Y. H. Jiang, J. L. Hua, H. Tian, and S. X. Qian, Optical limiting properties and ultrafast dynamics of six-branched styryl derivatives based on 1,3,5-triazine, J. Appl. Phys. 110(2011) 033518.

[14] Y. C. Wang, D. K. Zhang, H. Zhou, J. L. Ding, Q. Chen, Y. Xiao, S. X. Qian, Nonlinear optical properties and ultrafast dynamics of three novel boradiazaindacene derivatives, J. Appl. Phys. 108(2010) 033520.

[15] B. Li, R. Tong, R. Y. Zhu, F. S. Meng, H. Tian, S. X. Qian, The Ultrafast Dynamics and Nonlinear Optical Properties of Tribranched Styryl Derivatives Based on 1,3,5-Triazine, J. Phys. Chem. B 109(2005) 10705-10710. 